\title{
Ansiedade e Depressão entre Profissionais de Enfermagem em UPA durante a Pandemia da Covid-19
}

\section{Anxiety and Depression among Nursing Professionals at UPA during the Covid-19 Pandemic \\ La Ansiedad y Depresión entre los Profesionales de la Enfermería en la UPA durante la Pandemia de Covid-19}

\author{
Letícia Piffer \\ Maria Luiza Gana Schmidt ${ }^{1}$ \\ Universidade Estadual Paulista "Júlio de Mesquita Filho" (UNESP) \\ João Massuda Júnior \\ Instituto Federal de Mato Grosso do Sul (IFMS)
}

\begin{abstract}
Resumo
Estudo descritivo, correlacional, tipo transversal, que avaliou ansiedade e depressão entre profissionais de enfermagem de uma Unidade de Pronto Atendimento (UPA). Os dados foram coletados mediante um questionário sociodemográfico e a Escala Hospitalar de Ansiedade e Depressão (HADS). Participaram doze técnicos, onze auxiliares de enfermagem e sete enfermeiros, sendo a maioria do gênero feminino $(80,0 \%)$, casados $(40,0 \%)$ e com faixa etária predominante de 41 a 50 anos (36,7\%). Os respondentes apresentaram grau de ansiedade normal $(66,7 \%)$ e grau de depressão normal $(86,7 \%)$. As subescalas de ansiedade $(\alpha=0,71)$ e depressão $(\alpha=0,67)$ apresentaram adequada consistência interna. A análise de associação entre os escores totais referentes à avaliação de ansiedade e depressão indicou uma correlação estatisticamente significativa $(p=0,01)$, positiva e forte $(\rho=0,741)$. A HADS mostrou boa sensibilidade para avaliar sintomas de ansiedade e depressão, porém não evidenciou escores elevados no período da pandemia da covid-19 nos respondentes.

Palavras-chave: profissionais da enfermagem,; ansiedade, depressão, covid-19
\end{abstract}

\begin{abstract}
Descriptive, correlational, cross-sectional study, which evaluated anxiety and depression among nursing professionals at an Emergency Care Unit (UPA). Data were collected using a sociodemographic questionnaire and the Hospital Anxiety and Depression Scale (HADS). The study included twelve technicians, eleven nursing auxiliaries and seven nurses, most of them female (80.0\%), married (40.0\%) and with a predominant age group between 41 and 50 years old (36.7\%). The respondents showed regular anxiety (66.7\%) and depression (86.7\%) levels. The anxiety $(\alpha=0.71)$ and depression $(\alpha=0.67)$ subscales showed adequate internal consistency. The association analysis between the total scores of anxiety and depression assessment indicated a statistically significant $(p=0.01)$, positive and strong $(\rho=0.741)$ correlation. HADS indicated good sensitivity to assess anxiety and depression symptoms; however, it did not show high scores in the period of the covid-19 pandemic in respondents.
\end{abstract}

Keywords: nursing professionals, anxiety, depression; covid-19

\section{Resumen}

Estudio descriptivo, correlativo y transversal, que evaluó la ansiedad y la depresión entre los profesionales de enfermería de una Unidad de Atención de Emergencia (UPA). Los datos se recogieron usando un cuestionario sociodemográfico y la Escala Hospitalaria de Ansiedad y Depresión (HADS). Participaron doce técnicos, once auxiliares de enfermería y siete enfermeros, la mayoría de mujeres $(80,0 \%)$, casados $(40,0 \%)$ y con edad predominante entre 41 y 50 años $(36,7 \%)$. Los encuestados presentaron grados de ansiedad $(66,7 \%)$ y de depresión $(86,7 \%)$ normales. Las subescalas de ansiedad $(\alpha=0,71)$ y depresión $(\alpha=0,67)$ presentaron adecuada consistencia interna. El análisis de asociación entre las puntuaciones totales de la evaluación de ansiedad y depresión indicó una correlación estadísticamente significativa $(p=0,01)$, positiva y fuerte $(\rho=0,741)$. La HADS mostró buena sensibilidad para evaluar los síntomas de ansiedad y depresión, sin embargo, no evidenció puntuaciones elevadas en el período de la pandemia de covid-19 en los encuestados.

Palabras clave: profesionales de enfermería, ansiedad, depresión, covid-19

\footnotetext{
${ }^{1}$ Endereço para contato: Avenida Marechal Deodoro, 366, apto. 14, Assis, São Paulo. CEP: 198060-140. Telefone: (18) 3302-5896. E-mail: maria.Ig.schmidt@unesp.br
} 


\section{Introdução}

A Unidade de Pronto Atendimento (UPA) é um estabelecimento de saúde de complexidade intermediária situado entre a Atenção Básica de Saúde e a Atenção Hospitalar. É de encargo da unidade compor uma rede organizada de atenção às urgências, em conjunto com a Atenção Básica à Saúde e com a Rede Hospitalar. São estabelecimentos de saúde integrados ao Sistema Único de Saúde (SUS) que foram implantados em vários municípios do país, visando fortalecer a Política Nacional de Atenção às Urgências (Santos, 2015).

Foram constituídas para melhorar as articulações dos serviços de atenção à saúde da população estruturados no país em três níveis complementares: atenção básica; de média e alta complexidade. É, portanto, a principal via de acesso da população para o atendimento de urgências e emergências (Konder \& O’Dwyer, 2015).

Essas Unidades funcionam 24 horas e têm como objetivo concentrar os atendimentos de saúde de complexidade intermediária, compondo uma rede organizada em conjunto com a atenção básica, atenção hospitalar, atenção domiciliar e o Serviço de Atendimento Móvel de Urgência (SAMU) 192. Em termos de estrutura, a UPA oferece de forma simplificada raio-X, eletrocardiografia, pediatria, laboratório de exames e leitos de observação, propiciando à população melhoria no acesso de atendimento pelo Sistema Único de Saúde (Brasil, 2017).

A UPA é destinada a prestar o primeiro atendimento e estabilizar os pacientes acometidos por quadros agudos ou agudizados de natureza clínica e casos de natureza cirúrgica e de trauma, mediante investigação diagnóstica inicial e posterior conduta necessária para cada caso. Deste modo, mantém pacientes em observação, por até 24 horas, para elucidação diagnóstica ou estabilização clínica. Após este período, os pacientes que necessitam da continuidade do cuidado são encaminhados para internação em serviços hospitalares de retaguarda, por meio da regulação do acesso à assistência, para realização de procedimento de alta complexidade (Brasil, 2017; Cuduro \& Macedo, 2018).

Dentre os fatores responsáveis pela procura da população a estas unidades de emergência, estão: ". . . baixa oferta ambulatorial; ausência de procedimentos de triagem; falência da rede no que diz respeito à retaguarda de especialistas; reserva de leitos em unidades intensivas e; a não absorção pelo próprio hospital do atendimento gerado na emergência" (Oliveira, Pinel, Gonçalves, \& Diniz, 2013, p. 74). Outro fator também apontado pelos autores diz respeito à percepção dos usuários de que estes serviços, por serem dotados de recursos tecnológicos e humanos especializados, têm maior efetividade de respostas na resolução de seus problemas de saúde (Oliveira et al., 2013).

O quadro funcional das UPAs é constituído por uma equipe de médicos, farmacêuticos, técnicos de radiologia, enfermeiros, técnicos e auxiliares de enfermagem, profissionais administrativos, de vigilância, manutenção e zeladoria, sendo os profissionais da enfermagem os principais responsáveis por uma parcela significativa de intervenções aos usuários (Cuduro \& Macedo, 2018).

A dinâmica da organização do trabalho destes profissionais possui ritmo intenso em decorrência da alta demanda e, também, devido às situações a que estão expostos, tais como: realização de variadas tarefas ao mesmo tempo, atenção constante aos sinais de informações de máquinas ou equipamentos; e dependência do trabalho de outros colegas. Devido à intensificação do ritmo de trabalho, às vezes necessitam reduzir as pausas para refeições 
ou ficar até mesmo sem se alimentar (Gomes, 2014). Somado a isto, atuam em escalas rodiziantes em turnos diurnos e noturnos.

Trettene, Ferreira, Mutro, Tabaquim e Razera (2016) identificaram expressividade dos resultados sobre a incidência e caracterização do estresse em profissionais atuantes em UPAs, o que revela ser um contexto que merece ser investigado. $O$ impacto negativo referente à atuação de profissionais de enfermagem em Unidades de Emergência sobre a qualidade de vida também foi apontado por Kogien e Cedaro (2014).

Vale ressaltar que, ". . . os serviços públicos de emergência têm sofrido com a superlotação, o ritmo de trabalho acelerado, a sobrecarga dos profissionais de saúde e a precariedade de recursos físicos, humanos e materiais" (Veloso et al., 2016, p. 3970), expondo os profissionais de enfermagem a diversos estressores laborais e riscos à saúde física e mental.

Este contexto de exposição a diferentes riscos à saúde sofreu um enorme impacto na sua dinâmica a partir do dia 31 de dezembro de 2019, quando a Organização Mundial de Saúde (OMS) foi informada, na China, sobre casos de pneumonia de etiologia desconhecida na cidade de Wuhan, Província de Hubei. O agente causal da doença foi, posteriormente, denominado SARS-CoV-2 (Almeida, 2020).

De acordo com o Brasil (2020), o primeiro caso no Brasil foi confirmado no dia 26 de fevereiro e, em 3 de março, havia cerca de 500 casos suspeitos. Diante do aumento de casos, informado pela Secretaria Municipal da Saúde de São Paulo (São Paulo, 2020), somado à ausência de vacinas e de tratamento comprovadamente eficaz, a estratégia de distanciamento social foi apontada como a mais importante intervenção para o controle da doença (Teixeira et al., 2020).

No entanto, para as equipes de assistência à saúde, especialmente aqueles profissionais que estão no cuidado direto de pacientes com suspeita ou diagnóstico confirmado de covid-19 em serviços de atenção primária, nas unidades de pronto atendimento e nos hospitais, a recomendação de permanecer em casa não se aplica (Teixeira et al., 2020, p. 3466).

Surge então, no meio científico, um alerta sobre a situação da exposição dos profissionais da saúde tanto quanto ao risco de contaminação biológica, decorrente da alta carga viral recebida, como também dos impactos à saúde mental destes trabalhadores, devido ao estresse decorrente dos atendimentos dos pacientes em condições de trabalho inadequadas e com escassez de equipamentos de proteção individual (Almeida, 2020; Teixeira et al., 2020; Medeiros, 2020; Helioterio et al., 2020).

Em meio à pandemia, medidas de saúde pública preventivas junto à população em geral foram tomadas (Fillis et al., 2020; Sarti, Lazarini, Fontenelle, \& Almeida, 2020; Maciel et al., 2020).

As ações e estratégias foram apresentadas segundo eixos: Medidas de Saúde Pública Individuais, Comunitárias e Ambientais. Foram desenvolvidas ações de monitoramento, distribuição de máscaras, medidas ambientais de higiene e desinfecção de superfícies, testagem de profissionais e população, estruturação de unidades de atendimento, assistência psicológica, ações de vigilância epidemiológica, transporte sanitário e Equipamentos de Proteção Individuais (EPI) (Maciel et al., 2020, p. 24).

Contudo, diante da gravidade de exposição ao risco de adoecimento, a ". . . necessidade da proteção dos profissionais dos estabelecimentos de saúde ganhou merecido destaque, 
o mesmo não se verifica para outros grupos ocupacionais" (Jackson, Assunção, Algranti, Garcia, Saito, \& Maeno, 2020, p. 2).

Sendo um dos estabelecimentos de atendimento aos pacientes acometidos por covid-19, a UPA passou a ser um dos locais de extremo risco aos profissionais de saúde, especialmente aos da enfermagem, uma vez que a atuação desses/as trabalhadores/as é elemento central no enfrentamento da pandemia. Desse modo, além dos fatores de risco já existentes, esses profissionais passaram a receber mais esta sobrecarga de demanda no cotidiano laboral.

Estudos realizados em diferentes contextos têm apontado como os aspectos da organização do trabalho são fontes de estresse ocupacional que podem levar à exaustão emocional e produzem efeitos adversos à saúde dos profissionais de enfermagem, dentre os quais, fatores predisponentes à depressão e à ansiedade (Moura et al., 2018; Gomes \& Oliveira, 2013; Oliveira \& Pereira, 2012; Manetti, \& Marziale, 2007; Tselebis, Moulou, \& Ilias, 2001).

Embora a literatura aponte a existência de diversos estudos sobre a saúde das trabalhadoras de enfermagem que atuam em urgência e emergência, a maioria destes estão restritos às unidades hospitalares. Ainda hoje, são escassas as pesquisas realizadas especificamente em unidades não hospitalares, tais como as UPAs (Gomes, 2014).

De forma semelhante, não encontramos, em nossos estudos bibliográficos, resultados de pesquisas com indicadores de ansiedade e depressão em trabalhadores de enfermagem atuantes em UPAs, o que justifica a realização desta pesquisa, que teve como objetivo geral: avaliar a presença de ansiedade e depressão entre profissionais de enfermagem numa UPA no período da pandemia da covid-19.

\section{Método}

\section{Delineamento do Estudo}

Trata-se de um estudo descritivo, correlacional e de corte transversal. As características da amostra pesquisada, assim como os resultados dos instrumentos para diagnóstico de ansiedade e depressão, foram analisados por meio da utilização da estatística descritiva (distribuição de frequência). O Teste Exato de Fisher foi aplicado para a análise da associação entre depressão/ansiedade e as variáveis da pesquisa. Da mesma forma, foi verificada a correlação entre ansiedade e depressão por meio da utilização do coeficiente de correlação de Spearman.

A presente pesquisa seguiu as diretrizes propostas pelo Conselho Nacional de Saúde na Resolução n. 466, de 12 de dezembro de 2012, e foi aprovada pelo Comitê de Ética em Pesquisa com Seres Humanos da Plataforma Brasil, sob o número de parecer CAAE 25513519.6.0000.5401.

\section{Local da Pesquisa e Procedimentos da Coleta de Dados}

Esta pesquisa foi realizada numa UPA localizada num município do interior de São Paulo que possui aproximadamente 100 mil habitantes. Esta Unidade funciona de modo ininterrupto, nas 24 horas do dia e em todos os dias da semana, incluídos feriados e pontos facultativos. O contato com os participantes foi possibilitado pela coordenadora de enfermagem, sendo então realizada uma amostragem por conveniência, ou seja, foram incluídos os 
indivíduos trabalhadores que se interessaram em participar e que estavam prontamente disponíveis. A coleta de dados foi realizada numa sala administrativa da instituição com infraestrutura adequada.

Foram realizadas cinco visitas para coleta de dados, sendo duas no mês de junho e três no mês de agosto de 2020. O intervalo referente à pesquisa de campo se deu respeitando a fase laranja de flexibilização do município em relação à classificação da pandemia. Desde o início da pandemia, o município contabilizou 1.234 casos confirmados, correspondentes a 1,17\% da população. Foram confirmadas 23 mortes por covid-19, ou seja, uma taxa de letalidade de $1,86 \%$.

Os pesquisadores seguiram as orientações sanitárias de proteção à covid-19 e a presença na instituição ocorreu com os cuidados de uso de equipamentos de proteção individual e distanciamento de dois metros entre o pesquisador e o participante.

O formulário foi preenchido pelo pesquisador mediante as respostas emitidas pelo entrevistado.

A leitura e assinatura do Termo de Consentimento Livre e Esclarecido (TCLE) e o preenchimento da escala necessitou de, aproximadamente, quinze a vinte minutos e, de um modo geral, os itens foram bem compreendidos pelos respondentes.

\section{População e Amostra}

A amostra deste estudo foi composta por profissionais da equipe de enfermagem de uma UPA localizada num município do interior do estado de São Paulo, implantada no ano de 2014. No período de coleta de dados, o quadro funcional dos trabalhadores de enfermagem da Unidade era de 73 indivíduos no total, sendo 16 enfermeiros, 33 auxiliares de enfermagem e 24 técnicos de enfermagem. Desse total, participaram da pesquisa doze técnicos, onze auxiliares de enfermagem e sete enfermeiros.

\section{Instrumento de Coleta de Dados}

Para coleta de dados, foi utilizado um instrumento para levantamento dos dados sociodemográficos (idade, gênero, situação conjugal e escolaridade) e profissionais (categoria profissional, tempo de atuação na função, tempo de atuação na instituição, carga horária de trabalho semanal, presença de outro vínculo empregatício e remuneração recebida, segundo o salário mínimo vigente no momento da coleta dos dados) e a Escala Hospitalar de Ansiedade e Depressão (Zigmond \& Snaith, 1983), em sua versão traduzida e validada para a língua portuguesa (Botega, Pondé, Medeiros, Lima, \& Guerreiro, 1998).

Embora tenha sido inicialmente proposta para pacientes ambulatoriais na detecção de estados depressivos e de ansiedade, esta escala pode ser aplicada em diversos contextos (c), sendo que atualmente tem sido utilizada para diagnosticar ansiedade e depressão em pacientes psiquiátricos ou não psiquiátricos (Botega et al., 1998; Schmidt, Dantas, \& Marziale, 2011; Oliveira \& Pereira, 2012).

A Escala Hospitalar de Ansiedade e Depressão (HADS, do inglês Hospital Anxiety and Depression Scale) foi escolhida por ser de fácil e rápida aplicação e por ser constituída de variáveis de interesse (ansiedade e depressão). Esta escala é composta por 14 itens, sendo sete deles (1, 3, 5, 7, 9, 11 e 13) voltados para avaliação da ansiedade (HADS-A) e os outros sete (2, 
4, 6, 8, 10, 12 e 14), para a depressão (HADS-D). A subescala de ansiedade verifica a presença de sintomas de transtorno de ansiedade generalizada e a de depressão investiga a perda da capacidade de cuidar das tarefas diárias de vida (Guilland, Cruz, \& Kaszubowski, 2018).

A escala está estruturada em questões fechadas, pontuadas de zero a três, e aborda aspectos como sentir-se tenso ou contraído, ter o mesmo gosto pelas mesmas coisas de antes, sentir espécie de medo como se alguma coisa ruim fosse acontecer, dar risada e se divertir quando ver coisas engraçadas, estar com a cabeça cheia de preocupações, sentir-se alegre, ficar sentado à vontade e se sentir relaxado, estar lento para pensar e fazer coisas, ter sensação ruim de medo como um frio na barriga ou um aperto no estômago, perder o interesse em cuidar da aparência, sentir-se inquieto como se não pudesse ficar parado em lugar nenhum, ficar animado esperando as coisas que estão por vir, ter a sensação de entrar em pânico e sentir prazer quando assiste a um bom programa de televisão, de rádio ou quando ler alguma coisa.

Cada item é respondido numa escala original de quatro pontos, que vão de inexistente (0) a grave (3). O escore geral de ambas as subescalas varia de 0 a 21 . Ambas as doenças, ansiedade e depressão, foram classificados como normal (0-7 pontos), leve (8-10), moderada (11-15) e grave (16-21). Os valores mais elevados indicam níveis mais altos de ansiedade e depressão. É sugerido o valor 8 como ponto de corte, considerando os valores inferiores como ausência de ansiedade e depressão. As pontuações obtidas entre 8 e 10 para cada subescala poderão indicar uma possível perturbação clínica, e entre 11 e 21, uma provável perturbação clínica (Zigmond \& Snaith, 1983).

Neste estudo em específico, a análise do Alfa de Cronbach das subescalas relativas ao diagnóstico de ansiedade $(\alpha=0,71)$ e depressão $(\alpha=0,67)$ indicaram uma boa consistência interna destas subescalas, sendo que os resultados alcançados podem ser considerados semelhantes aos obtidos nos estudos desenvolvidos por Botega, Bio, Zomignani, Garcia e Pereira (1995), $\alpha=0,68$ para ansiedade e $\alpha=0,77$ para depressão.

\section{Análise dos Dados}

Utilizou-se estatística descritiva, teste de Correlação de Spearman e Teste Exato de Fisher para a avaliação da associação entre ansiedade e depressão e as demais variáveis pesquisadas neste estudo, tal qual sugerido por Kim (2017).

\section{Resultados}

A amostra foi composta por 30 profissionais da enfermagem, sendo 12 técnicos, 11 auxiliares e sete enfermeiros. Desses, $80 \%$ eram do gênero feminino e $20 \%$ masculino, a maioria casados(as) (40\%) e solteiros(as) (26,7\%), com idade entre 41 e 50 anos $(36,7 \%)$ e 21 e 30 anos $(26,7 \%)$.

Dentre os participantes, $73,3 \%$ informaram terem sido contratados por meio de contrato de trabalho formal pela CLT (contratados por uma fundação de ensino), 23,3\% disseram ter vínculo em regime estatutário (contratados pela Prefeitura Municipal) e um relatou ser autônomo com Cadastro Nacional de Pessoa Jurídica (CNPJ).

Quanto ao tempo de atuação na instituição, 50\% dos participantes informaram trabalhar na UPA por um período de três a seis anos. A carga horária de trabalho identificada foi de 
30, 36 e 40 horas semanais, realizadas em escalas rodiziantes. No que tange à remuneração, $63,3 \%$ apontaram receber de um a dois salários mínimos vigentes. Dentre os 30 participantes, dezesseis reportaram ter outro vínculo de trabalho.

Tabela 1

Distribuição de frequência referente à presença de ansiedade e depressão entre profissionais de enfermagem que atuam numa Unidade de Pronto Atendimento (UPA)

\begin{tabular}{|c|c|c|c|c|c|}
\hline \multirow{2}{*}{ Variável pesquisada } & \multicolumn{4}{|c|}{ Classificação } & \multirow[b]{2}{*}{ Total } \\
\hline & Normal & Leve & Moderada & Severa & \\
\hline Ansiedade $(\mathrm{N}=30)$ & $66,7 \%$ & $16,7 \%$ & $13,3 \%$ & $3,3 \%$ & $100,0 \%$ \\
\hline Depressão $(\mathrm{N}=30)$ & $86,7 \%$ & $13,3 \%$ & $0,0 \%$ & $0,0 \%$ & $100,0 \%$ \\
\hline
\end{tabular}

De acordo com os resultados da Tabela 1, observa-se que houve uma ocorrência elevada de participantes com escores abaixo de oito, tanto para ansiedade como para depressão, indicando grau de ansiedade e depressão normais para a maioria dos respondentes.

Ainda assim, foram identificados trabalhadores que reportaram um grau leve $(16,7 \%)$, moderado $(13,3 \%)$ e severo $(3,3 \%)$ para ansiedade e um grau leve $(13,3 \%)$ para depressão, sugerindo a necessidade de atenção, por parte da equipe responsável pela saúde ocupacional na unidade pesquisada, para com estes profissionais (Tabela 1).

Tabela 2

Distribuição de frequência referente à comorbidade de depressão leve e ansiedade entre profissionais de enfermagem que atuam numa Unidade de Pronto Atendimento (UPA)

\begin{tabular}{ccccc}
\hline \multirow{2}{*}{ Variável pesquisada } & \multicolumn{3}{c}{ Ansiedade - Classificação } & \multirow{2}{*}{ Total } \\
\cline { 2 - 4 } & Leve & Moderada & Severa & $100 \%$ \\
\hline Depressão leve $(\mathrm{N}=4)$ & $50,0 \%$ & $25,0 \%$ & $25,0 \%$ & $100 \%$ \\
\hline
\end{tabular}

Foi avaliada a hipótese de comorbidade entre depressão e ansiedade na amostra pesquisada, sendo encontrados quatro casos de participantes que reportaram simultaneamente depressão leve e algum grau de ansiedade (Tabela 2).

A análise de associação entre os escores totais referentes à avaliação de ansiedade e depressão indicou uma correlação positiva, estatisticamente significativa e forte (rô de Spearman $=0,741$ e $p$-valor $=0,01$ ) entre ambas as variáveis pesquisadas, não sendo possível, em virtude das características deste estudo, apontar o sentido da relação entre ambas.

A análise da associação entre ansiedade e as demais variáveis de interesse neste estudo não apontou a existência de associação estatisticamente significativa entre esta e as seguintes variáveis: sexo $(p=0,321)$, faixa etária $(p=0,579)$, estado civil $(p=0,333)$, escolaridade ( $p$ $=0,352)$, categoria profissional $(p=0,422)$, tempo de atuação na função $(p=0,643)$, tempo de atuação na instituição $(p=0,166)$, carga horária $(p=0,631)$, vínculo empregatício $(p=$ $0,901)$, outro vínculo empregatício $(p=0,146)$ e remuneração recebida $(p=0,795)$.

Ainda que não tenha sido identificada uma associação estatisticamente significativa entre as variáveis estudadas, foi observado, na amostra pesquisada, que indivíduos com idade entre 41 e 50 anos e 21 e 30 anos, separados e viúvos, com ensino superior completo e especialização, 
enfermeiros, que trabalhavam entre dois e quatro anos ou há mais de 20 anos na função em questão, que cumpriam uma carga horária semanal de 36 horas de serviço, servidores estatutários e que recebiam uma remuneração entre cinco e oito salários mínimos, apresentaram um maior grau de ansiedade quando comparados aos outros grupos pesquisados.

A análise da associação entre depressão e as demais variáveis de interesse neste estudo também não apontou a existência de associação estatisticamente significativa entre esta e as seguintes variáveis: sexo $(p=0,557)$, faixa etária $(p=1,000)$, estado civil $(p=0,073)$, escolaridade $(p=0,518)$, categoria profissional $(p=0,514)$, tempo de atuação na função $(p=0,440)$, tempo de atuação na instituição $(p=0,867)$, carga horária $(p=0,801)$, vínculo empregatício $(p=1,00)$, outro vínculo empregatício $(p=0,602)$ e remuneração recebida $(p=0,074)$.

Ainda que uma associação estatisticamente significativa não tenha sido identificada, é preciso destacar que, na amostra pesquisada, foi observada maior depressão entre indivíduos do sexo feminino, separados(as) e viúvos(as), com especialização, enfermeiros, que trabalham há mais de 20 anos na função em questão, cumprem uma jornada de 36 horas semanais e recebem entre cinco e oito salários-mínimos.

No que tange à categoria profissional, os enfermeiros pesquisados neste estudo apresentaram maior pontuação na classificação leve para depressão (28,6\%), quando comparados a técnicos $(9,1 \%)$ e auxiliares de enfermagem $(8,3 \%)$, que apresentaram resultados relativamente menores.

\section{Discussão}

O estudo revelou que, apesar de o trabalhador da enfermagem estar exposto a um ambiente de trabalho com situações estressantes, que exigem dele habilidade para seu enfrentamento e que podem gerar seu adoecimento físico e mental (Moura et al., 2018; Trettene et al., 2016; Gomes \& Oliveira, 2013; Oliveira \& Pereira, 2012; Manetti \& Marziale, 2007; Tselebis et al., 2001), a maioria dos trabalhadores pesquisados apresentou grau considerado normal para ansiedade.

De forma semelhante, ainda que diversas evidências científicas associem o ambiente de trabalho, os processos organizacionais, a sobrecarga quantitativa, qualitativa, emocional e cognitiva à presença de sintomas depressivos em profissionais de saúde, dentre eles os profissionais da área de enfermagem, mesmo atuando em uma UPA, os respondentes, na sua maioria, reportaram um grau normal para depressão.

Este panorama faz com que os achados desta pesquisa sigam de encontro aos resultados apresentados na literatura, uma vez que estudos realizados com enfermeiros, em outros contextos de trabalho, identificaram significativa prevalência de depressão entre os profissionais investigados (Vargas \& Dias, 2011).

Em atuantes na Estratégia Saúde da Família (ESF), por exemplo, foi identificado, na maioria dos participantes, a presença de sintomatologia depressiva, ainda que estes não se reconhecessem como doentes. Nestes estudos, os principais fatores associados ao sofrimento psíquico estavam relacionados às condições do trabalho (Fernandes \& Marcolan, 2017).

Ferreira e Ferreira (2015), em pesquisa de revisão sobre depressão em trabalhadores da enfermagem, também identificaram estudos $(n=7)$ que reconheceram algum grau de depressão ou comprometimento da saúde mental dos profissionais de enfermagem, sendo 
indicado que o adoecimento destes trabalhadores poderia estar associado a fatores internos e externos ao trabalho.

Estes autores concluíram ainda que a depressão é um problema de saúde que tem acometido com frequência os trabalhadores de enfermagem, porém alertaram para escassez de estudos relacionados ao tema entre os anos de 2000 e 2010 (Ferreira \& Ferreira, 2015).

Outro ponto de divergência identificado entre os resultados desta pesquisa e outros estudos realizados por outros pesquisadores é a menor presença de ansiedade entre os profissionais que reportaram possuir duplo vínculo de trabalho quando comparados àqueles que possuem apenas um vínculo empregatício. Schmidt et al. (2011), por exemplo, identificaram em seus estudos uma maior ocorrência de ansiedade em trabalhadores de instituições privadas ( $p=0,011$ ) e entre aqueles que tinham duplo emprego ( $p=0,027)$.

Em relação à presença de depressão entre os diferentes profissionais de enfermagem, os resultados apontaram que os enfermeiros participantes deste estudo apresentaram maior pontuação na classificação leve para depressão $(28,6 \%)$, quando comparados a técnicos $(9,1 \%)$ e auxiliares de enfermagem (8,3\%). De acordo com os autores, estes dados indicam um alinhamento dos resultados encontrados neste estudo com outras pesquisas anteriormente desenvolvidas.

Por fim, não encontramos na literatura estudos semelhantes para estabelecermos comparações diretas com os achados deste trabalho. As pesquisas que utilizaram a HADS em profissionais de enfermagem foram coletadas geralmente em outros contextos com características laborais diferentes, tais como unidades de saúde (Oliveira \& Pereira, 2012) e blocos cirúrgicos (Schmidt et al., 2011).

No entanto, a maioria dos profissionais de enfermagem apresentou escore para a medida de ansiedade e depressão inferior a oito, não sendo classificados como casos possíveis para a ansiedade e depressão, resultado semelhante aos obtidos no estudo de Schmidt et al. (2011) com profissionais de enfermagem em unidades de saúde.

\section{Considerações Finais}

Os resultados obtidos permitem proferir que o objetivo proposto foi atingido, isto é, conhecer os níveis de ansiedade e depressão em profissionais de enfermagem de uma UPA no período da pandemia.

A HADS mostrou boa sensibilidade para avaliar sintomas de ansiedade e depressão, porém não evidenciou escores elevados no período da pandemia da covid-19 nos respondentes. 0 pequeno número de sujeitos da amostra poderia ser responsável por esse achado, sendo considerado uma das principais limitações deste estudo o tamanho reduzido da amostra, por isto sugerimos sua replicação numa amostra maior.

Outra limitação se deu em decorrência das oscilações de flexibilização, provocadas pela pandemia, que restringiram o acesso dos pesquisadores à UPA como forma de preservação de sua própria saúde, uma vez que o local é considerado de alto risco de contaminação da covid-19.

Outra limitação se deu devido à singularidade do contexto de trabalho onde a pesquisa foi realizada, uma UPA com profissionais da área de enfermagem, cuja literatura mostra-se incipiente, dificultando as correlações com outros estudos. 
A utilização de um instrumento simples como o HADS é relevante para rastreamento de transtornos do humor que podem passar despercebidos entre os trabalhadores. No entanto, ressaltamos que o estudo possui limitações, pois as prevalências de ansiedade e depressão foram obtidas por meio de um instrumento de triagem, e não foram realizadas entrevistas psiquiátricas para confirmação do diagnóstico de ansiedade e/ou depressão.

Apesar das limitações aqui apresentadas, o estudo revela importante avanço para o conhecimento da enfermagem, à medida que contribui com a literatura nacional sobre a temática da ansiedade e depressão entre os trabalhadores desta categoria profissional, sobretudo diante da constatação de que, até o momento, os estudos no contexto de UPA são escassos.

Considerando as elevadas estimativas feitas pela OMS, sobre a ocorrência de depressão como causa de adoecimento nas próximas décadas, e as características das condições e organização de trabalho das UPAs, que as evidenciam como ambientes estressantes e repletos de fatores predisponentes à depressão e à ansiedade entre seus trabalhadores, reiteramos o alerta para a importância de estudos voltados para a elaboração de medidas preventivas neste ambiente de trabalho.

\section{Referências}

Almeida, I. M. (2020). Proteção da saúde dos trabalhadores da saúde em tempos de COVID-19 e respostas à pandemia. Revista Brasileira de Saúde Ocupacional, 45(17), 1-10. doi:https://doi.org/10.1590/scielopreprints.140

Botega, N. J., Bio, M. R., Zomignani, M. A., Garcia, C., Junior, \& Pereira, W. A. B. (1995). Transtornos do humor em enfermaria de clínica médica e validação de escala de medida (HAD) de ansiedade e depressão. Revista de Saúde Pública, 29(5), 359-363. doi:https:// doi.org/10.1590/S0034-89101995000500004

Botega, N. J., Pondé, M. P., Medeiros, P., Lima, M. G., \& Guerreiro, C. A. M. (1998). Validação da escala hospitalar de ansiedade e depressão (HAD) em pacientes epiléticos ambulatoriais. Jornal Brasileiro de Psiquiatria, 47(6), 285-289.

Brasil. Ministério da Saúde (2017). UPA 24h - Unidade de Pronto Atendimento. Governo Federal. Recuperado de https://www.gov.br/saude/pt-br/acesso-a-informacao/ acoes-e-programas/upa-24h-unidade-de-pronto-atendimento

Brasil. Ministério da Saúde (2020). Painel de casos de doença pelo coronavírus 2019 (COVID-19) no Brasil pelo Ministério da Saúde. Governo Federal. Recuperado de https:// covid.saude.gov.br/

Cuduro, F. L. F., \& Macedo, S. M. K. (2018). Evaluación del ambiente de trabajo entre profesionales de enfermería en una unidad de urgencia y emergencia. Enfermería Global, 17(50), 361-399. doi:http://dx.doi.org/10.6018/eglobal.17.2.283991

Fernandes, D. M., \& Marcolan, J. F. (2017). Trabalho e sintomatologia depressiva em enfermeiros da Estratégia de Saúde da Família. SMAD: Revista eletrônica saúde mental álcool e drogas, 13(1), 37-44. doi:https://doi.org/10.11606/issn.1806-6976.v13i1p37-44

Ferreira, L. A. L., \& Ferreira, L. L. (2015). Depressão no trabalho da enfermagem: Revisão de literatura. Universitas: Ciências da Saúde, 13(1), 41-48. doi:https://doi.org/10.5102/ucs. v13i1.2849 
Fillis, M. M. A., Dellaroza, M. S. G., Machado, R. A., Pelaquim, T. A. A., Coelho, V. V., Barbosa, V. C. A. A., . . Trelha, C. S. (2020). Saúde do trabalhador em tempos de COVID-19: a experiência do município de Londrina. APS em Revista, 2(2), 106-113. doi:https://doi. org/10.14295/aps.v2i2.97

Gomes, H. O. (2014). O trabalho e saúde das profissionais de enfermagem em urgência e emergência: Estudo de caso em uma Unidade de Pronto Atendimento no município do Rio de Janeiro. (Dissertação de mestrado, Escola Regional de Saúde Pública Sérgio Arouca, Fundação Oswaldo Cruz, Rio de Janeiro). Recuperado de https://www.arca.fiocruz.br/ handle/icict/24335

Gomes, R. K., \& Oliveira, V. B. (2013). Depressão, ansiedade e suporte social em profissionais de enfermagem. Boletim de Psicologia, 63(138), 23-33. Recuperado de http://pepsic. bvsalud.org/scielo.php?script=sci_arttext\&pid=S0006-59432013000100004

Guilland, R., Cruz, R. M., \& Kaszubowski, E. (2018). Propriedades psicométricas do inventário de fatores psicológicos de doenças relacionadas ao trabalho: Um estudo com trabalhadores de frigoríficos. Psico-USF, 23(3), 539-554. doi:https://doi. org/10.1590/1413-82712018230312

Helioterio, M. C., Lopes, F. Q. R. S., Sousa, C. C., Souza, F. O., Pinho, P. S., Sousa, F. N. F., \& Araújo, T. M. (2020). Covid-19: Por que a proteção da saúde de trabalhadores e trabalhadoras da saúde é prioritária no combate à pandemia? Trabalho, Educação e Saúde, 18(3), e00289121. doi:https://doi.org/10.1590/1981-7746-sol00289

Jackson, J. M., Filho, Assunção, A. A., Algranti, E., Garcia, E. G., Saito, C. A., \& Maeno, M. (2020). A saúde do trabalhador e o enfrentamento da COVID-19. Revista Brasileira de Saúde Ocupacional, 45(14), 1-3. doi:https://doi.org/10.1590/2317-6369ed0000120

Kim, H.-Y. (2017). Statistical notes for clinical researchers: Chi-squared test and Fisher's exact test. Restorative Dentistry \& Endodontics, 42(2), 152-155. doi:https://doi.org/10.5395/ rde.2017.42.2.152

Kogien, M., \& Cedaro, J. J. (2014). Public emergency department: The psychosocial impact on the physical domain of quality of life of nursing professionals. Revista Latino-Americana de Enfermagem, 22(1), 51-58. doi:https://doi.org/10.1590/0104-1169.3171.2387

Konder, M. T., \& O’Dwyer, G. (2015). As Unidades de Pronto-Atendimento na Política Nacional de Atenção às Urgências. Physis: Revista de Saúde Coletiva, 25(2), 525-545. doi:https:// doi.org/10.1590/S0103-73312015000200011

Maciel, A. M. S., Silva, J. D., Filho, Sousa, R. L., Matos, T. L., Lima, N. C. C., Ramalho, K. M. V. G., \& Pires Neto, R. J. (2020). Aplicação de medidas de saúde pública para o enfrentamento à COVID-19 no município de Russas-CE. Cadernos ESP/CE, 14(1), 24-29. Recuperado de https://cadernos.esp.ce.gov.br/index.php/cadernos/article/view/394

Manetti, M. L., \& Marziale, M. H. P. (2007). Fatores associados à depressão relacionada ao trabalho de enfermagem. Estudos de Psicologia, 12(1), 79-85. doi:https://dx.doi. org/10.1590/S1413-294X2007000100010

Medeiros, E. A.S. (2020). Aluta dos profissionais de saúde no enfrentamento da COVID-19. Acta Paulista de Enfermagem, 33, 1-4. doi:https://dx.doi.org/10.37689/acta-ape/2020edt0003 Moura, A., Lunardi, R., Volpato, R., Nascimento, V., Bassos, T., \& Lemes, A. (2018). Fatores associados à ansiedade entre profissionais da atenção básica. Revista Portuguesa de Enfermagem de Saúde Mental, (19), 17-26. doi:https://dx.doi.org/10.19131/rpesm.0198 
Oliveira, E. B., Pinel, J. S., Gonçalves, J. B. A., \& Diniz, D. B. (2013). Nursing work in hospital emergency units - psychosocial risks: A descriptive study. Online Brazilian Journal of Nursing, 12(1), 73-88. doi:https://doi.org/10.5935/1676-4285.20134046

Oliveira, V., \& Pereira, T. (2012). Ansiedade, depressão e burnout em enfermeiros: Impacto do trabalho por turnos. Revista de Enfermagem Referência, 3(7), 43-54. doi:https://dx.doi. org/10.12707/RIII1175

Santos, K. K. S. (2015). Efeito das Unidades de Pronto Atendimento (UPAs) na mortalidade por infarto agudo do miocárdio nas capitais e regiões metropolitanas do Brasil (Dissertação de mestrado, Centro de Ciências Sociais Aplicadas, Universidade Federal de Pernambuco, Recife). Recuperado de https://repositorio.ufpe.br/handle/123456789/15043

Sarti, T. D., Lazarini, W. S., Fontenelle, L. F., \& Almeida, A. P. S. C. (2020). Qual o papel da Atenção Primária à Saúde diante da pandemia provocada pela COVID-19? Epidemiologia e Serviços de Saúde, 29(2), e2020166. doi:https://dx.doi.org/10.5123/s1679-49742020000200024

São Paulo (Cidade) (2020). Boletim Diário Covid-19 no município de São Paulo de 23 de abril de 2020. Cidade de São Paulo. Recuperado de https://www.prefeitura.sp.gov.br/ cidade/secretarias/saude/vigilancia_em_saude/doencas_e_agravos/coronavirus/index. php? $\mathrm{p}=296672$

Schmidt, D. R. C., Dantas, R. A. S., \& Marziale, M. H. P. (2011). Ansiedade e depressão entre profissionais de enfermagem que atuam em blocos cirúrgicos. Revista da Escola de Enfermagem da USP, 45(2), 487-493. doi:https://doi.org/10.1590/ S0080-62342011000200026

Teixeira, C. F. S., Soares, C. M., Souza, E. A., Lisboa, E. S., Pinto, I. C. M., Andrade, L. R., \& Espiridião, M. A. (2020). A saúde dos profissionais de saúde no enfrentamento da pandemia de Covid-19. Ciência \& Saúde Coletiva, 25(9), 3465-3474. doi:https://doi. org/10.1590/1413-81232020259.19562020

Trettene,A.S., Ferreira,J.A. F., Mutro, M.E.G., Tabaquim, M. L. M., \&Razera, A.P.R.(2016). Estresse em profissionais de enfermagem atuantes em Unidades de Pronto Atendimento. Boletim - Academia Paulista de Psicologia, 36(91), 243-261. Recuperado de http://pepsic.bvsalud. org/scielo.php?script=sci_arttext\&pid=S1415-711X2016000200002

Tselebis, A., Moulou, A., \& Ilias, I. (2001). Burnout versus depression and sense of coherence: study of Greek nursing staff. Nursing \& Health Sciences, 3(2), 69-71. doi:https://doi. org/10.1046/j.1442-2018.2001.00074.x

Vargas, D., \& Dias, A. P. V. (2011). Prevalência de depressão em trabalhadores de enfermagem de Unidade de Terapia Intensiva: Estudo em hospitais de uma cidade do noroeste do Estado São Paulo. Revista Latino-Americana de Enfermagem, 19(5), 1114-1121. doi:https://doi. org/10.1590/S0104-11692011000500008

Veloso, L. U. P., Laurindo, L. M. B., Sousa, L. R. P., Veloso, C., Silva, F. J. G., Junior, \& Monteiro, C. F. S. (2016). Prevalência de ansiedade em profissionais de enfermagem de urgência e emergência. Revista de Enfermagem UFPE on line, 10(11), 3969-3976. doi:https://doi. org/10.5205/1981-8963-v10i11a11479p3969-3976-2016

Zigmond, A. S., \& Snaith, R. P. (1983). The Hospital Anxiety and Depression Scale. Acta Psychiatrica Scandinavica, 67(6), 361-370. doi:https://doi.org/10.1111/j.1600-0447.1983. tb09716.x 


\section{Sobre os autores:}

Letícia Piffer: Graduanda na Universidade Estadual Paulista em Franca (UNESP), Campus de Assis. Atualmente é Bolsista de Iniciação Científica na Fundação de Amparo à Pesquisa do Estado de São Paulo (FAPESP). E-mail: leticiapiffer95@gmail.com, Orcid: https://orcid.org/ 0000-0002-6576-0778

Maria Luiza Gana Schmidt: Doutora em Saúde Coletiva pela Universidade Estadual de Campinas (UNICAMP). Mestre em Psicologia pela Universidade Estadual Paulista Júlio de Mesquita Filho (UNESP). Graduada em Psicologia pela Unesp. Atualmente é Docente do Departamento de Psicologia Social da Faculdade de Ciências e Letras na UNESP, Campus de Assis. E-mail: mlschmidt@uol.com.br, Orcid: https://orcid.org/ 0000-0002-3296-7238

João Massuda Júnior: Doutor em Psicologia pela Universidade Católica Dom Bosco (UCDB). Mestre em Administração pela Universidade Federal de Mato Grosso do Sul (UFMS). Graduado em Administração pela UFMS. Atualmente é Docente de Administração do Instituto Federal de Mato Grosso do Sul (IFMS), Campus Campo Grande. E-mail: joao.massuda@ifms.edu.br, Orcid: https://orcid.org/0000-0003-0523-6285.

Recebido em: 26/10/2020

Última revisão: 27/01/2021

Aceite final: 03/03/2021 
